\title{
Failed extubation in a tertiary-level hospital intensive care unit, Pretoria, South Africa
}

\author{
L G Mogase, MB BCh, FCS (SA), MMed (Surg), Cert Vasc; M Z Koto, MB ChB, FCS (SA), FACS, PhD \\ Department of General Surgery, Sefako Makgatho Health Sciences University, Pretoria, South Africa \\ Corresponding author: L G Mogase (lgmogase@gmail.com)
}

\begin{abstract}
Background and objective. A prospective cohort study sought to measure the incidence and outcomes of failed extubation in Dr George Mukhari Academic Hospital intensive care unit (ICU), as well as to identify possible factors associated with failed extubation.

Methods. Data were collected over a 6-month period from 1 July 2015 to 31 December 2015. Pre-intubation parameters recorded on the data collection sheet included secretions, Glasgow Coma Scale (GCS), fluid balance, Tobin index, partial pressure of carbon dioxide $\left(\mathrm{pCO}_{2}\right)$, partial pressure of oxygen $\left(\mathrm{PaO}_{2}\right)$, comorbidities and weaning method.

Results. A total of 242 patients were enrolled over the 6-month study period. Of the 242 patients, 86 were excluded owing to pre-set exclusion criteria (death before extubation; tracheostomy before extubation; re-intubation $>72$ hours post extubation). An extubation failure rate of $16.7 \%$ ( $n=26)$ was observed. The incidence of ventilator-associated pneumonia in the failed extubation group was $19.23 \%$, whereas death was recorded in $42.31 \%$ of patients who failed extubation. The average length of ICU stay in the reintubated group was 11.58 days, and 4.04 days for successfully extubated patients. Only low GCS had a statistically significant impact on failed extubation: $p=0.0025$; odds ratio (OR) for low v. normal 5.13 (95\% confidence interval (CI) 1.78 - 14.79). Other predictor variables measured did not reach statistical significance. Weaning method: $p=0.3737$, OR for No T-piece v. T-piece 1.65 (95\% CI 0.547 - 4.976); comorbidities: $p=0.5914$, OR for two or more comorbidities v. no comorbidities 2.079 (95\% CI 0.246 - 17.539), no comorbidities v. single comorbidity 0.802 (95\% CI 0.211 - 3.043); fluid balance: $p=0.6625$, OR for negative v. positive fluid balance 0.571 ( $95 \%$ CI 0.170 - 1.916), OR for neutral v. positive fluid balance $<0.001(95 \% \mathrm{CI}<0.001->999.999)$; $\mathrm{pCO}_{2}: p=0.7510$, OR for high v. normal $\mathrm{pCO}_{2} 1.344$ (95\% CI 0.346 - 5.213), OR for low v. normal $\mathrm{pCO}_{2} 1.515$ (95\% CI 0.501 - 4.576); $\mathrm{PaO}$ : $p=0.4405$, OR for high v. normal 1.156 (95\% CI 0.382 - 3.494); OR for low v. normal $\mathrm{PaO}_{2} 2.638$ (95\% CI 0.553 - 12.587); Tobin index (Fischer's exact test): $p=0.7476$.

Conclusion. Low pre-extubation GCS is a predictor of failed extubation.

Keywords. extubation; incidence; intubation; ventilator; morbidity; mortality.
\end{abstract}

South Afr J Crit Care 2021:37(3):86-89. https://doi.org/10.7196/SAJCC.2021.v37i3.446

Contribution of the study. The study is a prospective observational study conducted in a high-volume referral hospital. It adds valuable scientific information to a growing body of data on the topic of extubation failure. It further reinforces the importance of extubation failure and the requirement for due diligence to be paid before a patient is extubated.

Failed extubation is defined as the need for re-intubation from the time of removal of the endotracheal tube to up to 72 hours following extubation. ${ }^{[1-4]}$ Other authors report an end period of 48 hours, ${ }^{[5-7]}$ and Girault et al.$^{[8]}$ defined an end period of up to a week.

The accepted incidence of failed extubation is $10-20 \%{ }^{[9]}$ Failed extubation rates $>20 \%$ may indicate an overaggressive approach to patient weaning, whereas failed extubation rates $<10 \%$ may indicate an overly cautious approach, leading to unnecessarily prolonged ventilation days. Importantly, failed extubation has been found to be an independent predictor of morbidity (ventilator-associated pneumonia) and mortality. ${ }^{[5,9,10]}$

Ventilator-associated pneumonia (VAP) and death occur at a frequency of $27 \%$ and $50 \%$, respectively. ${ }^{[9]}$ Ventilator dependence, increased hospital costs and prolonged length of hospital stay are other deleterious outcomes associated with re-intubation. ${ }^{[10]}$ Possible factors associated with failed extubation include presence of cardiorespiratory comorbidities, poor cough strength and excessive secretions. ${ }^{[9]}$ Other possible factors include neurological impairment, positive fluid balance, a high Acute Physiological Assessment and Chronic Health Evaluation (APACHE) score and new-onset sepsis. ${ }^{[1,6,7]}$

In the setting of Dr George Mukhari Academic Hospital (DGMAH), the incidence, outcomes and possible factors associated with failed extubation are not known. A study assessing these will provide valuable scientific information unique to our setting. The aim of this study was to determine the outcomes of failed extubation in patients admitted to the DGMAH intensive care unit (ICU).

\section{Methods \\ Ethics}

Written consent was obtained from all patients who participated in the study. Ethics clearance was obtained from the institutional review board in accordance with the Declaration of Helsinki (ref. no. SMUREC/M/13/2025: PG). 


\section{Setting and study design}

This was a prospective cohort study investigating outcomes of failed extubation in DGMAH ICU over 6 months (July 2015 to December 2015). The observational unit of interest was failed extubation and the secondary observational units were factors associated with failed extubation in the ICU of DGMAH.

DGMAH is a central academic hospital, affiliated to the Sefako Makgatho Health Sciences University in Northwest Pretoria, South Africa (SA), which serves a region encompassing 1.7 million people. The DGMAH ICU is a multipurpose ICU catering for a wide range of patient demographics and pathology.

The target population of interest was all patients admitted to the ICU for ventilatory support. The source of population of interest was all patients admitted to the DGMAH ICU section who fulfilled the ICU admission criterion. The patients who met the extubation failure criterion were identified. Failed extubation was defined as the need for re-intubation from the time the endotracheal tube is removed to 72 hours post extubation.

Pre-extubation parameters (partial pressure of carbon dioxide $\left(\mathrm{pCO}_{2}\right)$, partial pressure of oxygen $\left(\mathrm{PaO}_{2}\right)$, fluid balance, weaning method, Tobin index, cough strength, comorbidities, secretions, pre-extubation Glasgow Coma Scale (GCS)) were all recorded as predictor variables for failed extubation. Re-intubated patients' data were analysed for frequency of failed extubation and incidence of death and pneumonia following failed extubation.

\section{Outcomes after data collection \\ Primary outcome \\ - Incidence of failed extubation \\ Secondary outcomes \\ - Risk factors for failed extubation \\ - Outcomes of failed extubation \\ ○ VAP \\ - Length of stay (LOS) (ICU) \\ - Mortality (ICU)}

\section{Eligibility criteria}

- All intubated patients admitted to DGMAH ICU

- Re-intubation within 72 hours after planned or accidental extubation

\section{Exclusion criteria}

- Patients extubated and re-intubated due to a blocked or incorrectly placed endotracheal tube

- Patients who died before extubation

- Patients who had a tracheostomy in situ at ICU discharge were also excluded from the study

\section{Data collection}

The alertness (expressed as GCS), Tobin index, age, sex, $\mathrm{pCO}_{2}$ and $\mathrm{PaO}_{2}$ pre-extubation and presence of comorbidities were recorded as predictor values.

All clinical data were collected by the researcher from medical records on a daily basis. The demographic and covariate data collected included age, sex and diagnosis of each patient included in the study. Pre-extubation parameters recorded on the first data collection sheet included the GCS (measured out of 10, as speech is not included for intubated patient assessment), patient's comorbid illnesses, weaning method used, $\mathrm{pCO}_{2}$ and $\mathrm{PaO}_{2}$, fraction of inspired oxygen, positive end expiratory pressure, fluid balance (positive/negative), the Tobin index and the total number of days in ICU. All patients who failed extubation were recorded on the data collection sheet. The outcomes of failed extubation that were recorded included development of pneumonia, death and the total number of days in ICU.

\section{Sample size}

Using a $95 \%$ two-sided confidence interval (CI), a $5 \%$ precision would require 156 patients to show a $20 \%$ incidence or similar of failed extubation.

\section{Statistical analysis}

Using univariate analysis, continuous variables were analysed using Student's $t$-test and categorical variables were analysed using $\chi^{2}$ or Fisher's exact test. A univariate analysis and a logistic regression analysis were performed with re-intubation as dependent variable and comorbidities, GCS, pre-extubation $\mathrm{pCO}_{2}$, pre-extubation $\mathrm{PaO}_{2}$, preextubation fluid balance and weaning method as predictor variables. Odds ratios (ORs) and 95\% CIs were calculated to estimate the relative risk of failed extubation. The statistical analysis was performed by an independent statistician using SAS Release 9.2 (SAS, South Africa) or higher running under Microsoft Windows for a personal computer. A $p$-value $<0.05$ was considered statistically significant. The frequency of mortality and VAP was reported for patients who failed extubation.

\section{Results}

During the study period, 242 patients were admitted to ICU. Of these 156 met the eligibility criteria and were included in the study.

The mean age of patients included in the study was 37 years ( 2 months to 82 years). There was no statistically significant association between age and failed extubation (Table 1). Eighty-one male (52\%) and 75 (48\%) female patients were included in the study (Table 1). Gender did not influence incidence of extubation failure (Table 1). The incidence of failed extubation was $26 / 156$ (16.7\%).

\section{Predictor variables}

A univariate analysis and a logistic regression analysis were performed with re-intubation as dependent variable and comorbidities, GCS, preextubation $\mathrm{pCO}_{2}$, pre-extubation $\mathrm{PaO}_{2}$, pre-extubation fluid balance and weaning method as predictor variables. Cough strength was not analysed as it was not routinely assessed. Furthermore, there was great heterogeneity in the manner in which cough strength was assessed. Airway secretions were also excluded from the analysis because of the subjective nature of the assessment of airway secretions.

Using a logistic regression model that included all predictor variables stated above, only a GCS $<10$ independently predicted extubation failure (OR failed extubation is 5.1 (CI 1.8 - 14.8)).

Self-extubation/unplanned extubation occurred in 19\% of patients in the study population. Six patients $(20 \%)$ whose extubations were unplanned required re-intubation. Furthermore, nine patients (30\%) that had self-extubated had a GCS $<10$ (Table 2).

\section{Discussion and conclusion}

Our study aimed to assess the incidence of failed extubation in the DGMAH ICU. Outcomes of failed extubation (pneumonia, mortality) and possible predictors of failed extubation were assessed.

The incidence of failed extubation in the DGMAH ICU was found to be $16.7 \%$. Thille et al. ${ }^{[9]}$ suggested an expected failed extubation 
Table 1. Predictor variables for extubation failure and outcomes of failed extubation (mortality, VAP and LOS)

\begin{tabular}{|c|c|c|c|c|}
\hline Variable & All $(n)$ & Successful extubation $(n)$ & Failed extubation $(n)$ & $p$-value \\
\hline Age (mean), years & 37 & 33.4 & 36.9 & $0.4554^{*}$ \\
\hline Gender & & & & $0.1323^{*}$ \\
\hline Female & 75 & 66 & 9 & \\
\hline Male & 81 & 64 & 17 & \\
\hline Tobin index & & & & $0.7476^{*}$ \\
\hline$>105$ & 20 & 16 & 4 & \\
\hline$<105$ & 136 & 114 & 22 & \\
\hline \multicolumn{5}{|l|}{ Glasgow Coma Scale } \\
\hline $10 / 10$ & 131 & 116 & 15 & $0.0002^{*}$ \\
\hline$<10$ & 25 & 14 & 11 & $0.0025^{\dagger}$ \\
\hline Comorbidities & & & & $0.2067^{*}$ \\
\hline 2 or more comorbidities & 7 & 4 & 3 & \\
\hline Single comorbidity & 25 & 21 & 4 & \\
\hline No comorbidities & 124 & 105 & 19 & \\
\hline Weaning method & & & & $0.1810^{*}$ \\
\hline No T-piece & 102 & 82 & 20 & \\
\hline T-piece & 54 & 48 & 6 & \\
\hline Fluid balance & & & & $0.7052^{*}$ \\
\hline Negative & 40 & 35 & 5 & \\
\hline Neutral & 1 & 1 & 0 & \\
\hline Positive & 115 & 94 & 1 & \\
\hline Pre-extubation $\mathrm{PaO}_{2}$ & & & & $0.2436^{*}$ \\
\hline High & 93 & 78 & 15 & \\
\hline Low & 16 & 11 & 5 & \\
\hline Normal & 47 & 41 & 6 & \\
\hline Pre-extubation $\mathrm{pCO}_{2}$ & & & & $0.1090^{*}$ \\
\hline High & 25 & 18 & 7 & \\
\hline Low & 37 & 29 & 8 & \\
\hline Normal & 94 & 83 & 11 & \\
\hline VAP, $n / N(\%)$ & 5 & Not assessed & $5 / 26(19.2)$ & N/A \\
\hline Mortality, $n / N(\%)$ & 11 & Not assessed & $11 / 26(42.3)$ & N/A \\
\hline Length of ICU stay, days & 5.29 & 4.04 & 11.58 & $<0.0001^{*}$ \\
\hline
\end{tabular}

Table 2. Outcomes of self-extubation, $n$ (\%)

\begin{tabular}{lllll}
\multicolumn{2}{c}{ Glasgow Coma Scale } & & \multicolumn{2}{c}{ Re-intubation } \\
\cline { 5 - 5 } \cline { 5 - 5 } & $\mathbf{1 0}$ & & Yes & No \\
\hline $9(30)$ & $21(70)$ & & $6(20)$ & $24(80)$
\end{tabular}

rate of $10-20 \%$. Based on previous studies, it can be deduced that an overaggressive or overly cautious approach at patient weaning was avoided at DGMAH ICU.

In our study, VAP was diagnosed in $19.23 \%$ of patients who failed extubation. A mortality of $42.31 \%$ was recorded in patients who failed extubation. No comparison of VAP and mortality between the successfully extubated and the failed extubation groups was made. The literature reports morbidity (VAP) and mortality of $27 \%$ and $50 \%$, respectively. ${ }^{[9]}$ Frutos-Vivar et al. ${ }^{[11]}$ reported a mortality of $25 \%$ in patients requiring re-intubation following failed extubation. In the study, re-intubation was an independent predictor of mortality, with an OR of 5.18. ${ }^{[1]]} \mathrm{A}$ post hoc analysis of prospective data on 74 medical ICU patients reported a mortality of $42 \%$ in patients who failed extubation. ${ }^{[12]}$ Kilba et al. ${ }^{[13]}$ reported a mortality of $28.57 \%$ in re-intubated patients compared with $6.67 \%$ in successfully extubated patients in a paediatric ICU based in Cape Town, SA.
A number of possible predictors of failed extubation were studied (comorbidities, fluid balance, secretions, GCS, $\mathrm{pCO}_{2}$ and $\mathrm{PaO}_{2}$ preextubation).

A low pre-extubation GCS was the only statistically significant predictor of failed extubation $(p=0.0025)$ with OR of low v. normal $=5.13$ (95\% CI 1.78 - 14.79). Owing to low numbers of patients who self-extubated, a regression analysis could not be performed to examine the relationship between self-extubation and failed extubation. In a study by Tadie et al., ${ }^{[14]}$ impaired neurological status was directly associated with extubation failure. In addition to prematurity and dysmorphology, decreased level of consciousness was significantly associated with higher rates of failed extubation. ${ }^{[13]}$ Other studies, however, seem to suggest that neurosurgical patients with GCS $<9$ could be successfully extubated, provided that airway secretions were minimal. ${ }^{[14]}$

Airway secretions were not analysed in the study because of the subjective nature of assessment and reporting. Vallverdu et al. ${ }^{[7]}$ suggested that excess secretions were associated with higher failed extubation rates. The impact of cough strength and excess airway secretions on failed extubation remains an area of keen interest, with prospective randomised trials still lacking.

In a prospective observational study evaluating patients at risk of extubation failure, $34 \%$ of patients aged $>65$ with chronic cardiac or respiratory disease failed extubation compared with $9 \%$ extubation failure 
rate in other patients with no comorbidities. ${ }^{[12]}$ In our study, there was a trend to lower numbers of comorbidities being associated with successful extubation despite the finding not reaching statistical significance.

In a study by Thille et al., ${ }^{[2]}$ a positive fluid balance on the day before extubation was associated with an increased risk of extubation failure. Fluid balance was not shown to have a significant impact on extubation failure in our study. Studies have demonstrated that high baseline B-type natriuretic peptide (BNP) or a rise in BNP levels during a spontaneous breathing trial (SBT) can predict failure of weaning readiness test, postextubation respiratory distress or extubation failure. ${ }^{[1,5,6]}$ Diuretic therapy guided by BNP values may shorten weaning duration, suggesting that inducing a negative fluid balance may hasten extubation. ${ }^{[9]}$

Our study did not show an association between pre-extubation $\mathrm{pCO}_{2} /$ $\mathrm{PaO}_{2}$ and extubation success. This should, however, be interpreted with caution as multiple studies suggest the contrary. ${ }^{[9,15,16]}$ Our study was not a randomised controlled trial and most patients were extubated with a normal $\mathrm{pCO}_{2}$ and $\mathrm{PaO}_{2}$ as per DGMAH ICU protocol.

A recent study found loss of lung aeration as assessed by lung ultrasound during the SBT, suggesting lung de-recruitment predicted post-extubation respiratory distress better than BNP or echocardiography did. ${ }^{[9]}$ A minimal oxygenation threshold was found to be one of the key criteria used to select patients for extubation. ${ }^{[9]}$ A study showed that a P/F ratio of below 200 was associated with an increased risk of extubation failure in neurosurgical patients; however, most other studies found no differences in oxygenation between patients who failed extubation and those successfully extubated.

Hypoxaemia may not necessarily predict weaning outcomes, but hypercapnia can. One study found that hypercapnia $\left(\mathrm{pCO}_{2} \geq 44 \mathrm{mmHg}\right.$ ) during SBT was independently associated with extubation failure. ${ }^{[15]}$ Another study that included patients with high prevalence of chronic respiratory disorders found that a $\mathrm{pCO}_{2}>54 \mathrm{mmHg}$ during the SBT was independently associated with prolonged weaning and mortality. ${ }^{[16]}$

An SBT plays a critical role in assessing weaning readiness. ${ }^{[10]}$ An SBT is performed by placing the patient on minimal (continuous positive airway pressure (CPAP)/pressure support (PS)) or no support (T-piece). The proviso for performing an SBT is that the patient's initial pathological insult has been reversed, with minimal ventilator settings, normal GCS and normal blood gas parameters.

The study did not show a significant difference in weaning outcomes between patients weaned on T-piece and those weaned on minimal pressure support (CPAP/PS) $(p=0.3737)$.

In a study performed by Cabello and colleagues, the SBT on a T-piece v. low PS with or without positive end-expiratory pressure (PEEP) was compared in patients with heart failure and difficult weaning. ${ }^{[16]}$ Patient effort was much lower in the PS group than the T-piece group and decreased even further when PEEP was added. ${ }^{[17]}$ In the study by Cabello et al., ${ }^{[16]}$ an important illustration was that most patients succeeded the PS test, while all failed the T-piece test. This is important as CPAP/PS reduces afterload and may mask potential cardiopulmonary deficiencies that may be apparent on T-piece, thus increasing the risk of extubation failure.

It should, however, be noted that our study was underpowered in assessing differences in SBT with T-piece v. no T-piece as patients both with and without cardiopulmonary comorbidities were compared.

In conclusion, the rate of failed extubation in DGMAH ICU is acceptable compared with previous studies. The outcomes of failed extubation are deleterious with a VAP rate of $19.23 \%$ and mortality of $42.31 \%$ in our study. The study also showed a longer length of ICU stay for re-intubated patients. Low GCS pre-extubation was demonstrated to be a significant predictor of extubation failure. Further randomised controlled trails are required to assess impact of fluid balance, cardiopulmonary comorbidities, airway secretions and cough strength on extubation failure.

\section{Study limitations}

This was a non-randomised trial conducted in a single institution. The trial objective was to record outcomes of failed extubation but lacked comparison in morbidity and mortality between successfully extubated patients and patients who failed extubation. In assessing the impact of comorbidities on extubation failure, the sample sizes for both groups were small and unequal. As a result, the power of Fisher's test to have detected a significant difference between $30.8 \%$ and $19.2 \%$ is only $18 \%$. Cough strength and airway secretion assessment remains subjective in the setting of DGMAH ICU, with possible contamination of results due to nonstandardised assessment and reporting. For this reason, airway secretions were not analysed in our study. The study was unable to determine the relationship between self-extubation and failed extubation.

\section{Declaration. None.}

Acknowledgements. I would like to acknowledge the mentorship, guidance, scientific contribution of Prof. M Z Koto, in both the initiation and completion of study. Appreciation also to Prof. H Schoeman for the statistical analysis performed and Mr M E Mabitsela for technical editing of the study.

Author contributions. LGM: data collection and analysis, writing of manuscript. MZK: advisory and editing role.

Funding. None.

Conflicts of interest. None.

1. Epstein SK, Ciubotaru RL, Wong JB. Effect of failed extubation on the outcome of mechanical ventilation. Chest 1997;112(1):186-192. https://doi.org/10.1378/chest.112.1.186

2. Thille AW, Harrois A, Schortgen F, Brun Buisson C, Brochard L. Outcomes of extubation failure in medical intensive care unit patients. Crit Care Med 2011;39(12):2612-2618. https://doi.org/10.1097 ccm.0b013e3182282a5a

3. Frutos-Vivar F, Ferguson ND, Esteban A, et al. Risk factors for extubation failure in patients following a spontaneous breathing trial. Chest 2006;130(6):1664-1671. https://doi.org/10.1378/

4. Krinsley JS, Reddy PK, Iqbal A. What is the optimal rate of failed extubation? Crit Care 2012;16:111. https://doi.org/10.1186\%2Fcc11185

5. Estaban A, Alia I, Gordo F, et al., The Spanish Lung Failure Collaborative Group. Extubation outcome after spontaneous breathing trials with T-Tube or pressure support ventilation. Am J Respir Crit Care Med 1997;156(2):459-465. https://doi.org/10.1164/ajrccm.156.2.9610109

6. Estaban A, Alia I, Tobin MJ, et al., The Spanish Lung Failure Collaborative Group. Effect of spontaneous breathing trial duration on outcome of attempts to discontinue mechanical ventilation.

7. Vallverdu I, Calaf N, Subirana M, Net A, Benito S, Mancebo J. Clinical characteristics, respiratory mechanical parameters, and outcome of two hour T-piece trial in patients weaning from mechanical ventilation. Am J Respir Crit Care Med 1998;158(6):186-192. https://doi.org/10.1164/ ajrccm.158.6.9712135

8. Girault C, Bubenheim M, Abroug F, et al. Non-invasive ventilation and weaning in the chronic hypercapnic respiratory failure patients: A randomised multicenter trial. Am J Respir Crit Care Med 2011;184(6):672-679. https://doi.org/10.1164/rccm.201101-0035oc

9. Thille AW, Richard JCM, Brochard L. The decision to extubate in the intensive care unit: Concise clinical review. Am J Respir Crit Care Med 2013;187(12):1294-1302. https://doi.org/10.1164/ rccm.201208-1523ci

10. Macintyre N. Discontinuing mechanical ventilator support. Chest 2008;132(3):1049-1056. https:// doi.org/10.1378/chest.06-2862

11. Frutos-Vivar F, Esteban A, Apezteguia C, et al. Outcome of re-intubated patients after scheduled extubation. J Crit Care 2011;26(5):502-509. https://doi.org/10.1016/j.jcrc.2010.12.015

12. Epstein SK, Ciuboturu RL. Independent effects of etiology of failure and time to reintubation on the outcome for patients failing extubation. Am J Respir Crit Care Med 1998;158(2):489-493. https:// doi.org/10.1164/ajrccm.158.2.9711045

13. Kilba MC, Salie S, Morrow B. Abstract pcclb-37: Risk factors and outcomes of extubation failure in a tertiary multidisciplinary paediatric intensive care unit (PICU) in Cape Town, South Africa. Pediatr Crit Care Med 2018;19(6S):255. https://doi.org/10.1097/01.pcc.0000538122.29259.75

14. Tadie JM, Behm E, Lecuyer L, et al. Post-intubation laryngeal injuries and extubation failure: A fiberoptic endoscopic study. Intens Care Med 2010;36:991-998. https://doi.org/10.1007/s00134$010-1847-\mathrm{z}$

15. Sellares J, Ferrer M, Cano E, Loureiro H, Valencia M, Torres A. Predictors of prolonged weaning and survival during ventilator weaning in respiratory ICU. Intens Care Med 2011;37:775-784. https://doi org/10.1007/s00134-011-2179-3

16. Cabello B, Thille AW, Roche-Campo F, Brochard L, Gomez FJ, Mancebo J. Physiological comparison of three spontaneous breathing trials in difficult-to-wean patients. Intens Care Med 2010;36:1171-1179. https://doi.org/10.1007/s00134-010-1870-0

17. Ishaaya AM, Nathan SD, Belman MJ. Work of breathing after extubation. Chest 1995;107(1):204209. https://doi.org/10.1378/chest.107.1.204 